\title{
Study of Natural Convection in Fluid-saturated Porous Thermal Insulations with Multiple Inclined Diathermal Partitions
}

\author{
Jayesh S. Chordiya*, Ram Vinoy Sharma \\ National Institute of Technology Jamshedpur, Jamshedpur 831014, Jharkhand, India
}

Corresponding Author Email: jayesh.subhash@gmail.com

https://doi.org/10.18280/ijht.370115

Received: 24 April 2018

Accepted: 3 August 2018

\section{Keywords:}

natural convection, porous medium, diathermal partition, numerical scheme

\begin{abstract}
Natural convection fluid flow and heat transfer is considered within a fluid-saturated porous enclosure with inclined diathermal partitions at $135^{\circ}$. The primary objective of the paper is to attenuate the heat transfer rate across a differentially heated porous enclosure that is encountered in thermal insulations. This suppression is brought about by obstructing the flow with diathermal partitions, targeted to isolate two specific regions of the enclosure. The temperature distribution in porous enclosure with no partitions reveal that, there exists a high temperature gradient in the vicinity of lower half of hot face and upper half of cold face. Isolating these regions from the rest of enclosure is the physics behind employing multiple inclined partitions. Momentum equations are defined by Darcy model and the governing equations are discretized using Finite Difference Method (FDM) and solved by Successive Accelerated Replacement (SAR) scheme. Fluid flow and heat transfer analysis is performed for following parameters: modified Rayleigh number $\left(R a^{*}\right)$ from $10^{2}$ to $10^{3}$, partition ratio $(P R)$ from 0 to 1 , aspect ratio $(A R)$ as 1 . One of the main findings of this study is that, to suppress the natural convection effectively, the fluid flow along the lower half of the hot wall and upper half of the cold wall must be well obstructed or mechanically isolated as these regions bear high temperature gradient. Maximum reduction in average Nusselt number is obtained for $P R=0.8$ irrespective of the value of $R a^{*}$.
\end{abstract}

\section{INTRODUCTION}

Porous media, depending upon the application, may serve to enhance or suppress the heat transfer across it. Applications like cooling in electronic packages, packed bed reactors are examples of heat transfer enhancement while thermal insulation, solar energy collection involve attenuating the heat transfer. The present study concerns with suppression of natural convection heat transfer across a differentially heated porous enclosure. A detailed literature based on convection in porous media can be found in the works of Bear, Greenkorn and Neild and Bejan [1-3]. Natural convection fluid flow and heat transfer within a fluid-saturated porous medium having internal obstructions, which may be conducting or insulated, has been the focus of research since past decade. These researches have been driven, predominantly, by the early works like those of Bejan and Anderson, Jang and Chen and Bejan who showed that internal obstructions within a differentially heated porous enclosure, could considerably attenuate the rate of heat transfer across it [4-6]. Tasnim et al. showed that employing an obstruction in a cavity is comparable to reducing $D a$ of the porous medium [7]. Jang and Chen, studied the effects of off-center diathermal partition and inclination angle of porous enclosure on natural convection heat transfer [5]. At lower $R a$ values partition location is found more influential than that at higher $R a$ values. The least value of Nusselt number is obtained at centrally positioned partition and at roughly $30^{\circ}$ inclination angle. Vasseur and Wang, studied the effect of a thermal barrier in a vertical porous layer separated by $N$ equally spaced partitions
[8]. The Nusselt number, in the boundary layer regime, has been found to vary inversely with $(1+N)^{4 / 5}$ while the greatest reduction in heat transfer, in the intermediate regime, has been obtained when the partition is located centrally. Varol and Oztop and Varol et al. analyzed a 2D solution of natural convection in solid adiabatic thin fin connected to fluidsaturated porous right triangular enclosures $[9,10]$. Varol et al. illustrated the effects of inclinedly inserted conductive thin plate on natural convection flow in a cavity filled with a porous medium [11]. They showed that heat transfer and flow field are strongly depended on position of the plate and was found to be higher for $135^{\circ}$ inclined plate.

Studies mentioned hitherto were based on Darcy model. Hsiao and Chen considered Brinkman-Forchheimer extended Darcy flow model to study a steady natural convection about a heated corrugated plate inserted in an enclosed porous medium useful in casting engineering [12]. Sathiyamoorthy and Narasimman studied the effect of the length and position of the fin on modification of the re-circulating cells and isotherms within the porous enclosure using the BrinkmanForchheimer-extended Darcy model [13]. Tasnim et al. considered non-Darcy model without the Forchheimer term to study free convection through a porous medium which was differentially heated from side with internal flow obstructions [7]. Both the aforementioned studies revealed that average Nusselt number falls as the length of the fin increases for all locations. The overall heat transfer rate can be managed with an appropriate selection of the fin position and length. Fewer studies related to partitions in porous media are available in last five years. Handful among them are the works of Ziad who 
numerically investigated transient magneto-hydrodynamic natural convection in partitioned square enclosure filled with a fluid-saturated porous medium with internal heat generation [14]. Rees et al. and Rees illustrated convection in a horizontal fluid-saturated porous layer heated from below and divided it into a number of similar sublayers by impervious and infinitesimally thin horizontal diathermal partitions $[15,16]$. Chordiya and Sharma have reported the effects of several kinds of orientations of internal partitions within a differentially heated porous enclosure [17].

A new physics that is being brought out in the present study is the way of obstructing the convection current, precisely, by orienting the diathermal partition to isolate two specific regions on the porous enclosure. The temperature distribution in a differentially heated porous enclosure reveal that in the vicinity of lower half of hot face and upper half of cold face, there exists high temperature gradient which leads to higher convection heat transfer, particularly, in these two regions. This can also be noticed from the higher values of local Nusselt number along these parts of the wall. Thereby, in order to suppress the natural convection heat transfer within the enclosure as a whole, these two regions must be specifically dealt with. The current study introduces a novel way to isolate these regions by employing multiple inclined partitions across the lower half of hot face and upper half of cold face. Besides, to the authors' best knowledge, such a study is yet to appear in the literature.

The objective of the present study is to suppress the natural convection fluid flow and heat transfer across a differentially heated square porous enclosure with multiple diathermal inclined partitions attached in it. The effect of position of partitions is studied and an optimum value of partition ratio has been found out that yields lowest average Nusselt number. Darcy model is considered with Boussinesq approximation to account for density variation. The whole point of such a model is sought to contribute in the redesign of a thermal insulations to effectively attenuate the convection heat transfer rate across it.

\section{MATHEMATICAL FORMULATION}

Figure 1 shows the schematic diagram of the model under study with geometric details and coordinate system. The fluidsaturated square porous enclosure has length $L$. Left and right walls are maintained at temperature $T_{h}$ and $T_{c}$ respectively, such that $T_{h}>T_{c}$. The top and bottom wall are insulated. Two diathermal partitions inclined at $135^{\circ}$ are attached on southwest and northeast regions of the enclosure. The end points of either of the partitions are at distance $D$ from origin and from farthest point from origin respectively. The study is confined to two-dimensional flow, since in the post-critical range of Darcy-modified Rayleigh number $\left(R a^{*}\right)$ the convection pattern is expected to be two-dimensional at first, Rees [17]. The flow is assumed to be steady, laminar and incompressible. The porous medium is assumed to be isotropic, homogenous and also that it is in local thermal equilibrium with the saturated fluid. Thermophysical properties of air are kept constant, except for density $\rho$. Density is assumed to change with temperature $T$ according to Boussinesq approximation. A Darcy model is considered without the Forchheimer term, since the current study is focused primarily on analyzing a natural convection flow in a porous cavity and not on convective boundary layer flow over the surface of a body in a high porous media, as was discussed in detail by Bejan and Poulikakos [18].

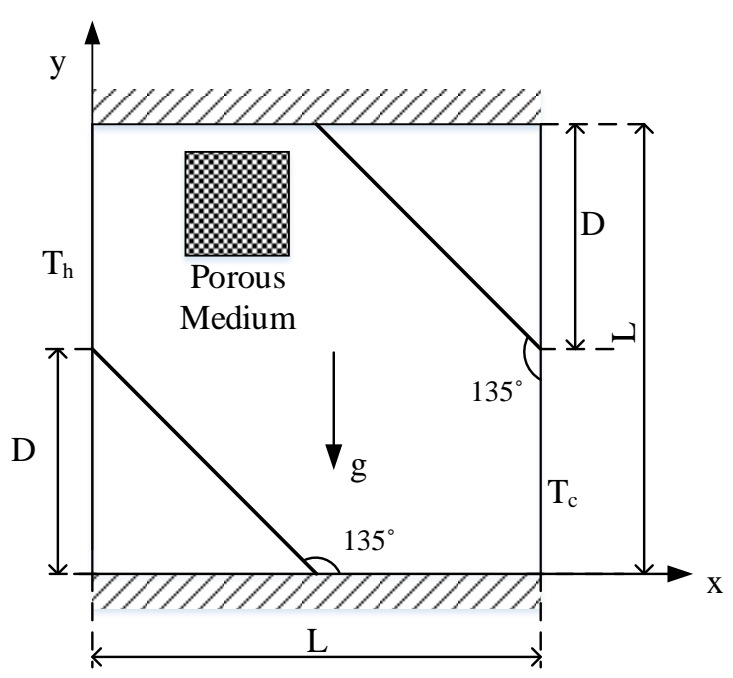

Figure 1. Schematic diagram of the model

Mindful of these assumptions, the governing equations for conservation of mass, momentum and energy for solid and porous medium in dimensional form can be written as,

$$
\begin{aligned}
& \frac{\partial u}{\partial x}+\frac{\partial v}{\partial y}=0 \\
& u=-\frac{K}{\mu} \frac{\partial p}{\partial x} \\
& v=-\frac{K}{\mu}\left(\frac{\partial p}{\partial x}+\rho g\right) \\
& \left(\rho c_{p}\right)_{f}\left(u \frac{\partial T}{\partial x}+v \frac{\partial T}{\partial y}\right)=k_{p}\left(\frac{\partial^{2} T}{\partial x^{2}}+\frac{\partial^{2} T}{\partial y^{2}}\right)
\end{aligned}
$$

These equations are subjected to following boundary conditions,

Left wall $(x=0,0 \leq y \leq L): \quad u=v=0, T=T_{h}$

Right wall $(x=L, 0 \leq y \leq L): \quad u=v=0, T=T_{c}$

Bottom and Top wall $(0 \leq x \leq L, y=0, L): u=v=0, \partial T / \partial y=0$

The matching conditions at diathermal partitions,

Lower Partition $(x+y=D): \quad u=v=0, \quad \partial T / \partial n^{-}=\partial T / \partial n^{+}$ Upper Partition $(x+y=2 L-D): u=v=0, \quad \partial T / \partial n^{-}=\partial T / \partial n^{+}$

Following dimensionless variables are used to nondimensionalize above equations and boundary conditions,

$$
\begin{aligned}
& X=\frac{x}{L} ; \quad Y=\frac{y}{L} ; \quad U=\frac{u}{\alpha_{e} / L} ; \quad V=\frac{v}{\alpha_{e} / L} ; \\
& \theta=\frac{T-T_{c}}{T_{h}-T_{c}}
\end{aligned}
$$

Here, effective thermal conductivity is given by, $\alpha_{e}=\frac{k_{p}}{\left(\rho c_{p}\right)_{f}}$ Following dimensionless parameters are used while nondimensionalizing above equations and boundary conditions, 
$R a^{*}=\frac{K g \beta \Delta T H}{v \alpha_{e}} ; P R=\frac{D}{L}$

Velocities are represented in terms of stream function $(\psi)$. The relation is given as,

$U=\frac{\partial \psi}{\partial Y} ; \quad V=-\frac{\partial \psi}{\partial X}$

Using the above relations along with dimensionless variables and parameters, Eq. (1)-(6) can be rewritten in nondimensional stream function and vorticity formulation as,

$\frac{\partial^{2} \psi}{\partial X^{2}}+\frac{\partial^{2} \psi}{\partial Y^{2}}=-R a^{*} \frac{\partial \theta}{\partial X}$

$\frac{\partial \psi}{\partial Y} \frac{\partial \theta}{\partial X}-\frac{\partial \psi}{\partial X} \frac{\partial \theta}{\partial Y}=\frac{\partial^{2} \theta}{\partial X^{2}}+\frac{\partial^{2} \theta}{\partial Y^{2}}$

These equations are subjected to following boundary conditions,

Left wall $(X=0,0 \leq Y \leq 1): \quad \psi=0, \quad \theta=1$

Right wall $(X=1,0 \leq Y \leq 1): \quad \psi=0, \quad \theta=0$

Bottom and Top wall $(0 \leq X \leq 1, Y=0,1): \quad \psi=0, \partial \theta / \partial Y=0$

The matching conditions at diathermal partitions,
Lower Partition $(X+Y=P R)$ :
$\psi=0, \quad \partial \theta / \partial n^{-}=\partial \theta / \partial n^{+}$
Upper Partition $(X+Y=2-P R)$
$\psi=0, \quad \partial \theta / \partial n^{-}=\partial \theta / \partial n^{+}$

The above equations are numerically solved and the results are analyzed qualitatively as well as quantitatively. Qualitative outcomes are visualized using streamlines and isotherms. On the other hand, quantitative outcome is analyzed by estimating the Nusselt number, local $(N u)$ as well as average $\left(N u_{a v g}\right)$.

$$
\begin{aligned}
& N u_{h, c}=-\left.\frac{\partial \theta}{\partial X}\right|_{X=0,1} \\
& N u_{a v g}=\int_{0}^{1} N u_{h, c}(Y) d Y
\end{aligned}
$$

\section{NUMERICAL METHOD}

The coupled differential governing equations are converted into algebraic equations using Finite Difference Method (FDM) and solved by Successive Accelerated Replacement (SAR) scheme. Central differencing with second-order accuracy is used to discretise governing equations, while second order forward and backward differencing is employed at the wall boundaries. The stream function and temperature have been solved using SAR scheme for all inner grid points. A suitable value of accelerating factor $(\omega)$ is chosen based on the accuracy obtained with regards to number of iterations required to attain convergence. Local Nusselt number along hot and cold wall are estimated and average Nusselt number is calculated by numerically integrating them using Simpson's $1 / 3^{\text {rd }}$ rule.

\subsection{SAR scheme}

Sinha and Sharma, Mishra et al. and Chordiya and Sharma have illustrated the applicability of the SAR scheme for solving system of partial differential equations in the study of two-dimensional natural convection fluid flow and heat transfer in porous media [19-23]. The basic idea of this scheme is to guess the profile for each transport property that satisfies the boundary conditions. If $\lambda$ is a transport property and the error in a typical governing equation at a grid point $(i, j)$ at $n^{\text {th }}$ iteration is $\tilde{\lambda}_{i j}^{n}$. Then, $(n+1)^{\text {th }}$ approximation of the variable $\lambda$ is obtained as,

$\lambda_{i j}^{n+1}=\lambda_{i j}^{n}-\omega \frac{\tilde{\lambda}_{i j}^{n}}{\partial \tilde{\lambda}_{i j}^{n} / \partial \lambda_{i j}^{n}}$

The accelerating factor, $\omega$, varies from 0 to 2 . The criterion set for convergence of stream function at all inner grid points is described as below. Value of $\varepsilon$ is the error tolerance limit with a small positive value.

$\frac{\sum_{\mathrm{i}=2}^{\text {imax }-1} \sum_{\mathrm{j}=2}^{j \max -1}\left|\lambda_{i j}^{\mathrm{n}+1}-\lambda_{i j}^{\mathrm{n}}\right|}{\sum_{i=2}^{\text {imax-1}} \sum_{j=2}^{j \max -1}\left|\lambda_{i j}^{n+1}\right|}<\varepsilon$

The important feature of this method is that the corrected value of the variable is immediately used upon becoming available. A numerical code based on this method is developed to solve the governing equations along with prescribed boundary conditions.

\section{RESULTS AND DISCUSSION}

A numerical analysis has been performed to access the natural convection fluid flow and heat transfer in fluidsaturated porous enclosure with multiple inclined partitions for following parameters: $10^{2} \leq R a^{*} \leq 10^{3} ; \quad 0 \leq P R \leq 1$. The influence of position of partition within the enclosure is evaluated by noticing streamlines and isotherms and convection heat transfer is estimated by calculating the value of average Nusselt number. An in-house computational code is written to solve the current numerical problem. Pertaining to the present configuration, the present code has been subjected to two validation checks; by comparing it with studies of, first, natural convection in plain porous enclosure and second, natural convection in porous enclosure with single inclined partition by Varol et al. [11]

\subsection{Validation of code for porous enclosure without partition}

Table 1. Comparison of average Nusselt number with results from literature for $P R=0$ (Porous enclosure)

\begin{tabular}{lrll}
\hline Authors & $\boldsymbol{R a}^{*} \mathbf{: 1 0}$ & $\mathbf{1 0 0}$ & $\mathbf{1 0 0 0}$ \\
\hline Walker and Homsy [24] & & 3.09 & 12.9 \\
Bejan [25] & & 4.20 & 15.80 \\
Manole and Lage [26] & & 3.11 & 13.63 \\
Moya et al. [27] & 1.06 & 2.80 & \\
Baytas and Pop [28] & 1.07 & 3.16 & 14.06 \\
\hline Present Study & 1.07 & 3.16 & 13.09 \\
\hline
\end{tabular}

Firstly, the code is compared against the benchmark solutions of Walker and Homsy, Bejan, Manole and Lage, Moya et al., Baytas and Pop [22-26]. It is a classical natural convection problem in a differentially heated square porous 
cavity. Table 1 shows the comparison with similar parameters and boundary conditions which are: Left wall is hot, right wall is cold, top and bottom are insulated. Here, value of $P R$ in code is set to 0 .

\subsection{Validation of code for porous enclosure with diathermal partition}

Secondly, to authenticate the code against study of Varol et al. [11] which is based on natural convection in porous enclosure with diagonal partition. It consists of a single diagonal partition inclined at $135^{\circ}$. Table 2 shows the comparison of results of present code with that of work in literature. Here, value of $P R$ in code is set to 1 .

The numerical comparison between the results obtained from present computational code agrees well in accordance with the results presented in literature. Thus, the code can be endorsed to study the problem stated in the current paper with greater assurance.

Table 2. Comparison of results with Varol et al. for $P R=1$ [11]

\begin{tabular}{ccc}
\hline $\boldsymbol{R a}^{*}$ & Varol et al. [11] & Present Study \\
\hline 100 & 1.601 & 1.633 \\
\hline 250 & 2.581 & 2.552 \\
\hline 500 & 3.771 & 3.831 \\
\hline 1000 & 5.449 & 5.731 \\
\hline
\end{tabular}

The results predicted in current paper are independent of grid size. Uniform structured mesh is generated and results are checked for grid size $41 \times 41$ to $261 \times 261$. It was found that for $R a^{*}<500$, grid size of $61 \times 61$ gave satisfactory results while that for values greater than 500, grid size of $241 \times 241$ were considered. The grid generation has been a typical challenge since it is obligatory that there must exist grid points along the inclined partitions. The matching conditions along the partition were solved by II-order forward and backward FDM. However, the forward and backward difference is with respect to normal direction along the partition. The foregoing section discusses the streamlines and isotherms for different positions of partition viz., $P R=0$ to 1 and $R a^{*}=100$ to 1000 .

\subsection{Effect of presence of partition on natural convection fluid flow and heat transfer}

This section discusses the effect of mere presence of multiple inclined partitions within a fluid-saturated square porous enclosure (Figure $2 \mathrm{~b}, P R=0.5$ ) on fluid flow by comparing the streamlines, isotherms, maximum absolute stream function value, local and average Nusselt number with those of porous enclosures without any partitions (Figure 2a, $P R=0)$. The streamlines reveal the flow obstruction, while isotherms help to notice the change in temperature gradient, particularly, in the two areas under concern i.e., in the vicinity of the lower half of hot face and upper half of cold face. The negative value of stream function indicate that flow is in anticlockwise direction. As seen clearly in Figure 2(a), the isotherms are not only concentrated more in the southwest and northeast regions of the enclosure but are also very step, indicating higher temperature gradient and hence higher heat transfer rate. This is because, in the southwest corner (lower half of hot face) the colder fluid comes in contact with hot face, gets heated and rises upwards; as it rises upwards its temperature keeps on increasing thereby decreasing the temperature difference between wall and fluid. The maximum temperature difference is thus, mostly, in the lower half of the hot face. Hence, the temperature gradients are steep in the vicinity of lower half of hot face. Next, this warmer fluid moves on towards the cold wall via the top face of the enclosure. It comes in contact with cold face on the upper half part first, gradually cools and move downwards; its temperature decreases and thus the temperature difference between the cold wall and fluid decreases. Thus, maximum temperature difference, in this case, is in the upper half part of cold face which can be clearly seen from the isotherm plots of Figure 2(a).

There are two facts worth noticing while comparing Figure 2(a) and Figure 2(b); first, number of innermost stagnant portion of streamlines and second, temperature gradient near southwest and northeast corners. There is only one inner stagnant portion of streamline in porous enclosure with no partition, which suggests that the fluid, as a whole is in motion which increases its momentum. However, this big rotund stagnant portion is broken up into four different small portions in four different parts of the enclosure, thereby distributing the share of momentum into four parts. Thus, suppressing the natural convection fluid flow effectively. As seen clearly in Figure 2(a), the isotherms are very steep in southwest and northeast corners on account of the reason mentioned above. However, this steepness is seen to considerably decrease in Figure 2(b). This is because, the inclined partition near the hot wall is also slightly heated up which causes the fluid in the complete vicinity to pre-heat before coming in contact with the hot wall. Thus, the temperature difference between hot wall and fluid is significantly less than in the former case. Consequently, the slope of temperature gradient drops.
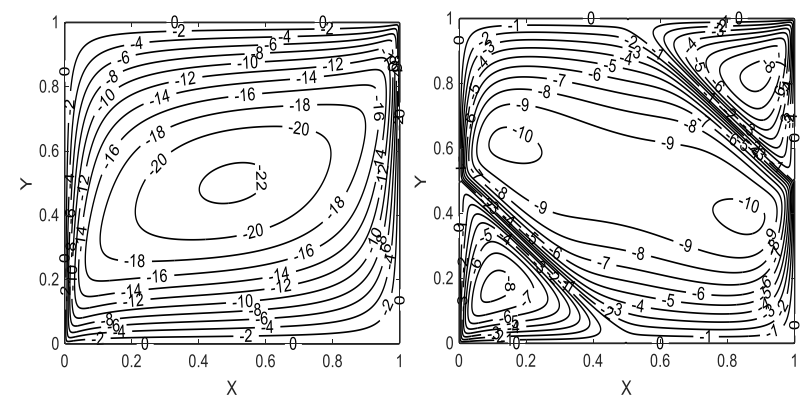

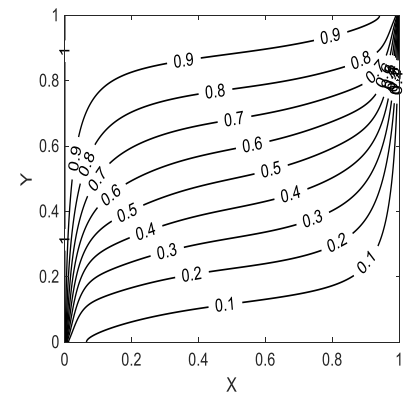

(a)

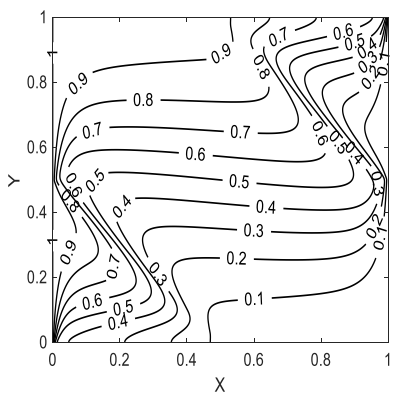

(b)
Figure 2. Streamlines (up) and isotherms (down) for square porous enclosure with: (a) no partition $(P R=0)$; (b) inclined partitions $(P R=0.5) ; R a^{*}=1000$

The magnitude of stream function signifies the strength of convection fluid flow. The gradient of stream function is velocity of fluid. The value of maximum absolute stream function for Figure 2(a) and Figure 2(b) is 22.296 and 10.217 
respectively. The drop in $|\psi|_{\max }$ is $54.18 \%$ which is very significant, to say the least. Hence, the objective of suppressing the natural convection fluid flow is thus served very effectively by employing the inclined partition. Figure 3 shows the variation of local Nusselt number along hot and cold face for fluid-saturated porous enclosure with and without partitions for $R a^{*}=1000$ and $P R=0$ and 0.5 .

It is evident, yet again, from the Figure 3 that there is a substantial drop of about $50 \%$ in the value of Nusselt number along the lower half of hot wall and upper half of cold wall, in the case of porous enclosure with multiple inclined partitions. The spike in the curve at $Y=0.5$ is due to the contact of partition since $P R=0.5$. There is cross-flow of fluid across the partition; fluid below the partition is moving downwards as it is relatively cooler while fluid above the partition is moving upwards as it is relatively warmer. This creates a temperature difference across the partition and hence generates the spike in the curve at $\mathrm{Y}=0.5$.

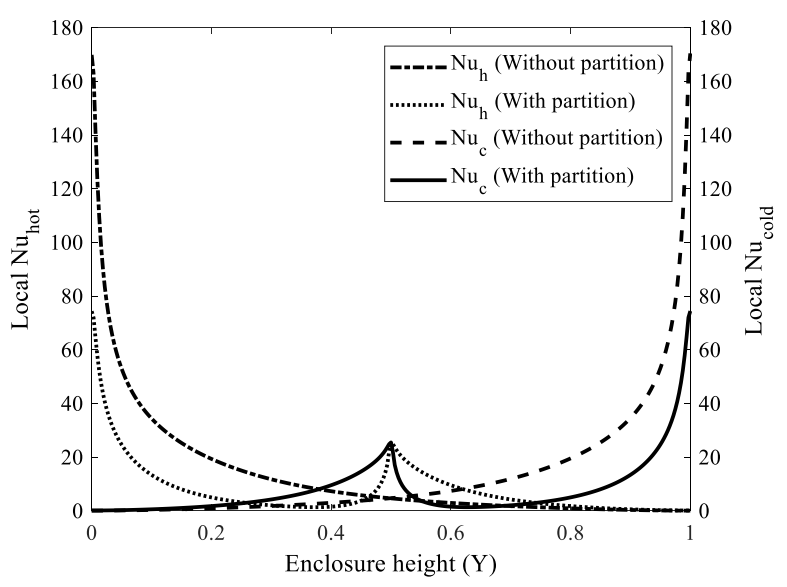

Figure 3. Local $N u$ on hot \& cold wall for enclosure with $(P R=0.5)$ and without $(P R=0)$ inclined partitions. $\left(R a^{*}=1000\right)$

The discussion hitherto has shown that convective fluid flow has been effectively obstructed by the presence of inclined partition. The influence of multiple inclined partition on convection heat transfer can be manifested by observing the values of average $N u$ which is illustrated in Figure 4. The variation of average $N u$ is shown for values of Darcy-modified Rayleigh number from $10^{2}$ to $10^{3}$ for porous enclosure with $(P R=0.5)$ and without $(P R=0)$ multiple inclined partitions.

It is clear from Figure 4 that average Nusselt number drops to about $50 \%$ due to presence of multiple inclined partition. As value of $R a^{*}$ increases, the strength of buoyancy increases which aids in the ease of convection flow, hence the value of Nusselt number increases in both the cases. It is also noted that the drop in $\mathrm{Nu}$ values is slightly more for higher values of $R a^{*}$.

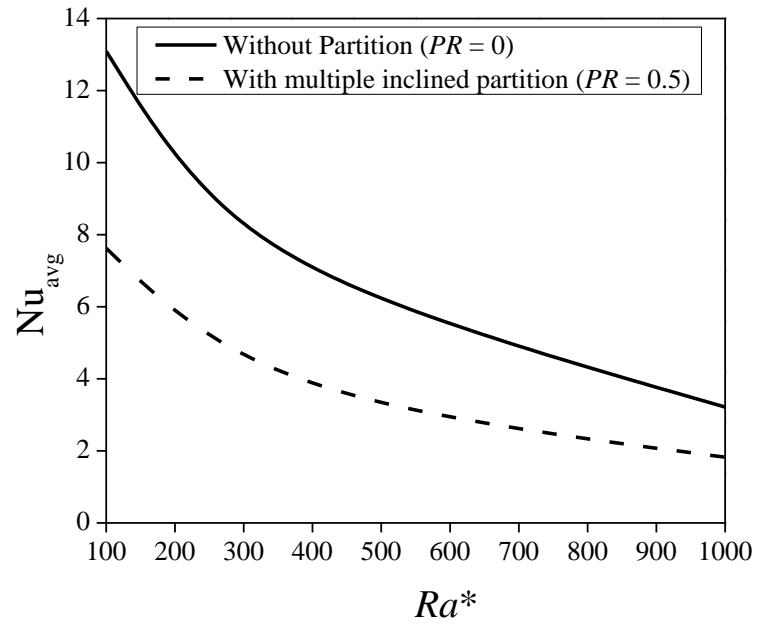

Figure 4. Average $N u$ for enclosure with $(P R=0.5)$ and without $(P R=0)$ inclined partitions

The purpose of this section was to access the effect of mere presence of the multiple inclined partition on convective fluid flow and heat transfer in fluid-saturated porous enclosure, which was performed by comparing streamlines, isotherms, maximum absolute stream function, local and average Nusselt number values with that of porous enclosure without any partitions. It is clear from all the aspects that employing the multiple inclined partition very substantially suppresses the convection fluid flow as well as heat transfer. Now, it becomes obligatory to evaluate the effect of position of partitions and to estimate the value of partition ratio $P R$ for which least Nusselt number is obtained. This is discussed in detail in the forthcoming section.

\subsection{Effect of position of partition on natural convection fluid flow and heat transfer}

This section discusses the effect of position of multiple inclined partitions within a fluid-saturated square porous enclosure on fluid flow as well as heat transfer by noticing the streamlines, isotherms, maximum absolute stream function, local and average Nusselt number values for partition ratio $P R$ from 0 to 1 . Value of $P R=0$ indicates enclosure without any partition and $P R=1$ indicates a single diagonal partition. Figure 5 demonstrates streamlines and isotherms for fluidsaturated square porous enclosure with multiple inclined partitions for $P R=0.5,0.8$ and 0.9 and $R a^{*}=1000$. The range of $P R$ is chosen such that the deviations in streamlines and isotherms are clearly noticeable. For any further low values of $P R$, the deviations become very ambiguous to notice. However, the effect of lower values of $P R$ is demonstrated later in discussion of its influence on Nusselt number.
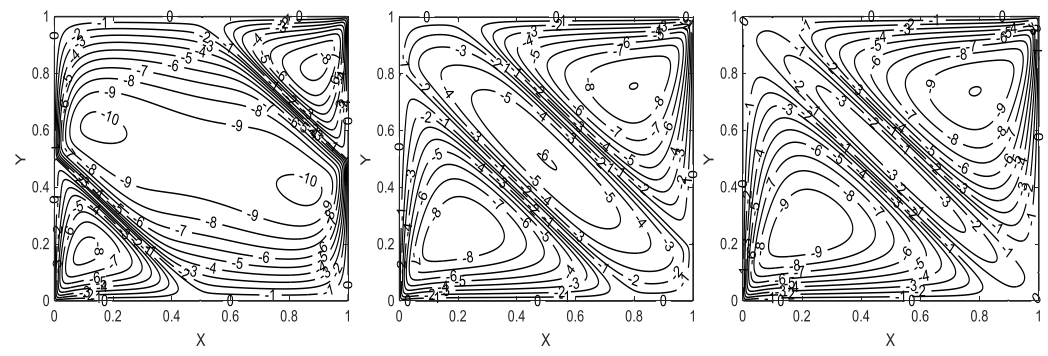


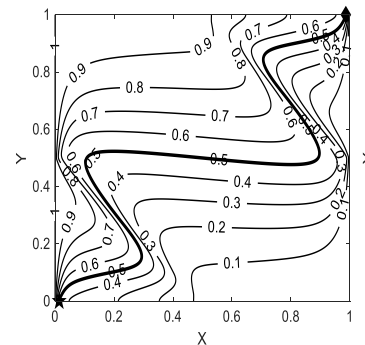

(a) $|\psi|_{\max }=10.217$

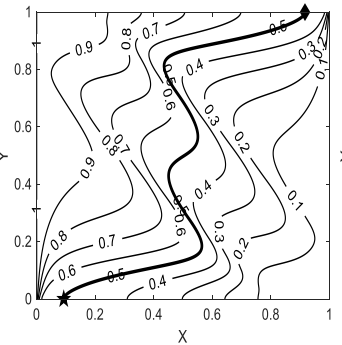

(b) $|\psi|_{\max }=9.0094$

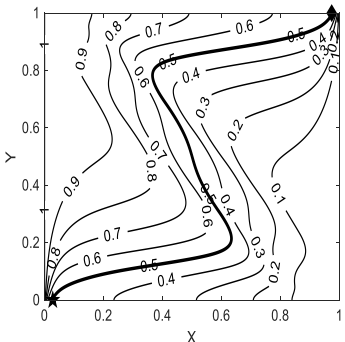

(c) $|\psi|_{\max }=10.0185$

Figure 5. Streamlines (up) and isotherms (down) for square porous enclosure with:

(a) $P R=0.5$; (b) $P R=0.8$; (b) $P R=0.9$. $\left(R a^{*}=1000\right)$

The central stagnant portion of streamlines are broken into multiple stagnant portions as was seen previously in Figure 2. In case $(a)$, there are four small stagnant portions at four different locations of enclosure such that those in the middle block are comparatively larger in size. The major portion of enclosure is acquired by the central block. The variation of absolute stream function value in the central block is approximately $1 \leq|\psi| \leq 10$ with maximum absolute stream function $\left(|\psi|_{\max }\right)$ value of 10.217 . In case $(b)$, the stagnant portions are broken into three equal sized portions at three different locations. The enclosure is acquired by all the blocks almost equally. The variation of absolute stream function value in the central and corner blocks is approximately $1 \leq|\psi| \leq 6$ and $1 \leq|\psi| \leq 8$ respectively with $|\psi|_{\max }$ value of 9.009 . While that in case $(c)$, out of three broken stagnant portions, the ones in the corner are comparatively larger in size. The major portion of enclosure is acquired by the corner blocks. The variation of absolute stream function value in the corner blocks is approximately $1 \leq|\psi| \leq 9$ with $|\psi|_{\max }$ value of 10.0185 . Thus, for $P R=0.8$ not only the variation in stream function values, but also the maximum absolute value of stream function is found to be the least of all. Temperature distribution is shown by isotherm contours. Here, the contour level of $\theta=0.5$ is highlighted for comparison between the three cases. Let this contour be termed as contour ' $C_{0.5}$ '. The end points of $C_{0.5}$ are marked with a pentagram and a diamond shaped marker. As seen in case $(a), C_{0.5}$ starts at $(X, Y)$ co-ordinates of $(0.01,0)$ and ends at $(0.98,1)$ which is very close to hot and cold wall respectively. In the central portion, it can be seen to extend horizontally from very near of hot wall to very near of cold wall. Also, the temperature gradient is high near the hot wall $(0.1 \leq X \leq 0.3$ and $0.1 \leq Y \leq 0.5)$ and near the cold wall as well $(0.7 \leq X \leq 0.9$ and $0.5 \leq Y \leq 0.9)$. In case $(b), C_{0.5}$ starts at $(X, Y)$ coordinates of $(0.1,0)$ and ends at $(0.91,1)$ which is comparatively farther to hot and cold wall respectively, as compared to case $(a)$. In the central portion, it can be seen to extend vertically by maintaining equal distance from hot and cold wall. Also, the temperature gradient is very low near the hot wall $(0.1 \leq X \leq 0.5$ and $0 \leq Y \leq 0.1)$ and near the cold wall as well $(0.5 \leq X \leq 0.9$ and $0.9 \leq Y \leq 1)$. In case $(c), C_{0.5}$ starts at $(X, Y)$ co-ordinates of $(0.09,0)$ and ends at $(0.91,1)$ which is comparatively farther to hot and cold wall respectively, as compared to case $(a)$. In the central portion, it can be seen to extend vertically with slight inclination towards hot and cold wall. Also, the temperature gradient is low near the hot wall $(0.1 \leq X \leq 0.6$ and $0 \leq Y \leq 0.2)$ and near the cold wall as well $(0.6 \leq X \leq 0.9$ and $0.8 \leq Y \leq 1)$ which is slightly greater than case (b) but lower than case $(a)$. Therefore, the temperature gradient near the hot wall and cold wall is found to be the least for $P R=0.8$.

The above discussion shows that natural convection fluid flow is certainly influenced by the position of partition and maximum obstruction is obtained for $P R=0.8$. The effect of partition ratio on convective heat transfer can be manifested by noticing the local and average Nusselt number. Nusselt number signifies the strength of convective heat transfer relative to conductive heat transfer. Figure 6 shows the variation of local Nusselt number along hot wall (Figure 6a) and cold wall (Figure 6b) for fluid-saturated square porous enclosure with $P R=0.5,0.8$ and 0.9 and $R a^{*}=1000$.

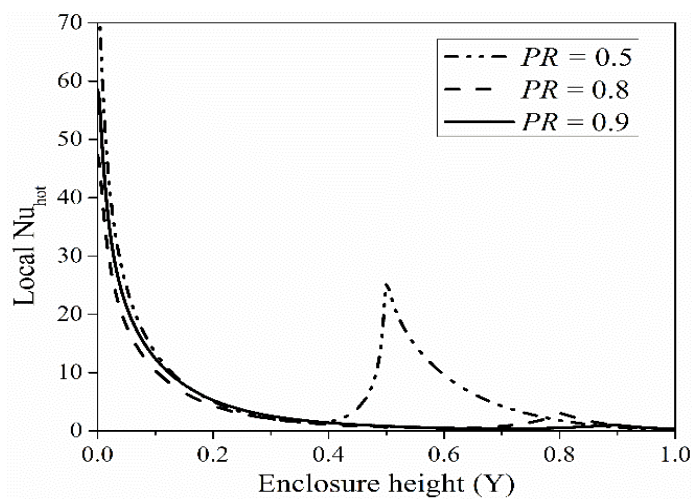

(a)

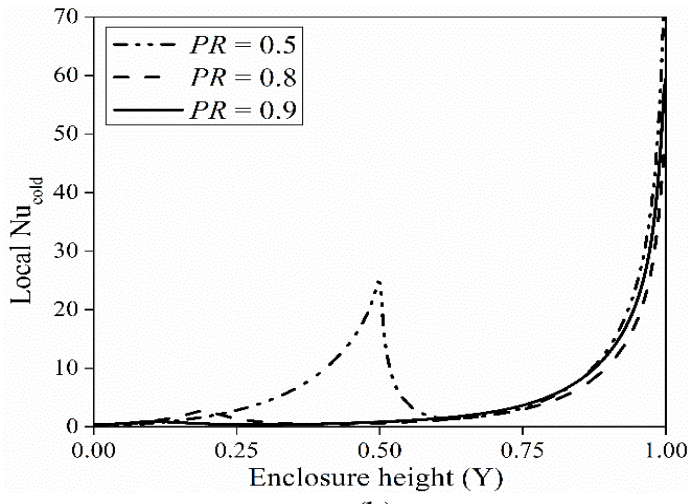

(b)

Figure 6. Local Nusselt number on (a) hot and (b) cold wall for enclosure with multiple inclined partitions. $\left(\mathrm{Ra}^{*}=1000\right)$

The spike in the curves of Figure 6 are due to presence of edges of partition at that particular enclosure height. The spikes are exactly seen to occur at $Y=0.5,0.8$ and 0.9 which are the values of $P R$. It is clear from the graph that lowest value of local Nusselt number is obtained for $P R=0.8$. The difference can be noticed over the lower half of hot face and upper half of cold face where value of Nusselt number significantly high. However, the relative values of $N u$ are lowest for $P R=0.8$. The reason can be given on the basis of distribution of isotherms previously explained. The temperature gradient near hot wall 
and cold wall for $P R=0.8$ is relatively lower than that for other two cases. Figure 7 depicts the variation for average Nusselt number against a wide range of $P R^{*}$ plotted for $R a=100,250$, 500 and 1000 .

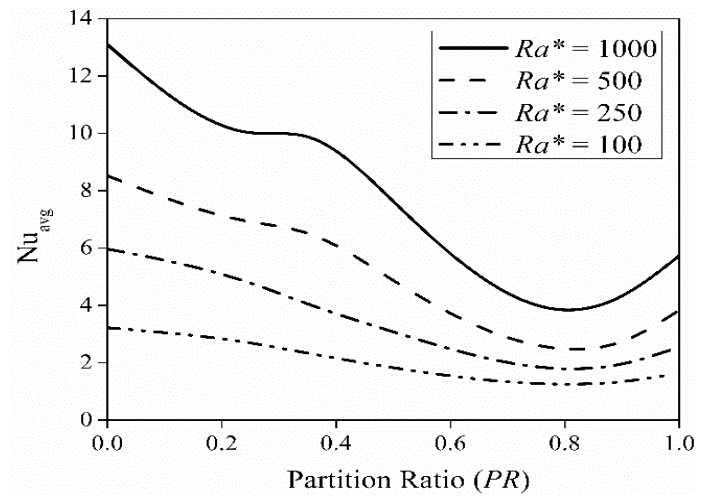

(a)

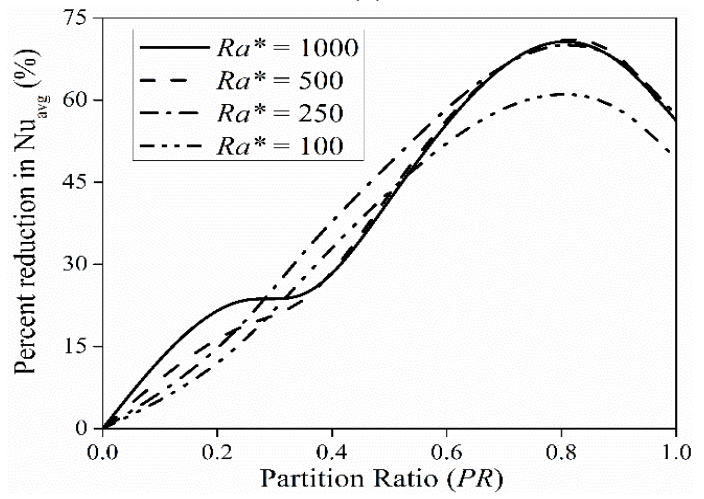

(b)

Figure 7. (a)Variation of $N u_{\text {avg }}$; (b) Percent reduction in $N u_{\text {avg }}$ with varying partition ratio for different values of $R a^{*}$

A comprehensive summary of above discussions about convective fluid flow and heat transfer in fluid-saturated square porous enclosure with multiple inclined partitions can be viewed in Figure 7. It can be clearly seen from Figure 7(a) that average Nusselt number increases with increase in value of $R a^{*}$ for all values of $P R$. Further, the least value of average Nusselt number is obtained for $P R=0.8$ irrespective of the value of $R a^{*}$. The percent decrease in convective heat transfer is up to $71 \%$ when $P R=0.8$. This is a significantly high reduction in the value of $N u_{\text {avg. }}$.

\section{CONCLUSIONS}

The present numerical study was based on suppressing the convective fluid flow and heat transfer in a fluid-saturated square porous enclosure with multiple inclined diathermal partitions attached to it. The effect of mere presence of partitions and its position in the enclosure was studied by evaluating streamlines, isotherms, maximum absolute stream function, local and average Nusselt number values for Darcymodified Rayleigh number $\left(R a^{*}\right) 100$ to 1000 and partition ratio $(P R) 0$ to 1 . Following conclusions may be drawn from the analysis performed above,

1. To suppress the natural convection fluid flow and heat transfer, the regions near the vicinity of lower half of hot face and upper half of cold face must be well obstructed or isolated from the rest of enclosure on account of high temperature gradient generating in these regions.
2. Mere presence of partition brings about substantial reduction in values of average Nusselt number.

3. The reduction in Nusselt number is more pronounced for higher values of Darcy-Modified Rayleigh number.

4. The maximum in Nusselt number is about $70 \%$ obtained for $P R=0.8$ for higher values of Darcy-modified Rayleigh number.

\section{REFERENCES}

[1] Bear J. (1972). Dynamics of fluids in porous media. Elsevier, New York.

[2] Greenkorn RA. (1983). Flow phenomena in porous media: Fundamentals and applications in petroleum, water and food production. CRC Press.

[3] Nield DA, Bejan A. (2006). Convection in porous media (Vol. 3). New York: springer.

[4] Bejan A, Anderson R. (1981). Heat transfer across a vertical impermeable partition imbedded in porous medium. International Journal of Heat and Mass Transfer 24(7): $\quad 1237-1245 . \quad$ https://doi.org/10.1016/00179310(81)90173-3

[5] Jang JY, Chen CN. (1989). Natural convection in an inclined porous enclosure with an off-center diathermal partition. Wärme-und Stoffübertragung 24(2): 117-123. https://doi.org/10.1007/BF01786445

[6] Bejan A. (1983). Natural convection heat transfer in a porous layer with internal flow obstructions. International Journal of Heat and Mass Transfer 26(6): 815-822. https://doi.org/10.1016/S0017-9310(83)801057

[7] Tasnim SH, Mahmud S, Dutta A. (2013). Energy streamlines analyses on natural convection within porous square enclosure with internal obstructions. Journal of Thermal Science and Engineering Applications 5(3): 031008. https://doi.org/10.1115/1.4023603

[8] Vasseur P, Wang CH. (1992). Natural convection heat transfer in a porous layer with multiple partitions. Chemical Engineering Communications 114(1): 145-167. https://doi.org/10.1080/00986449208936020

[9] Varol Y, Oztop HF. (2009). Control of buoyancyinduced temperature and flow fields with an embedded adiabatic thin plate in porous triangular cavities. Applied Thermal Engineering 29(2-3): 558-566. https://doi.org/10.1016/j.applthermaleng.2008.03.018

[10] Varol Y, Oztop HF, Varol A. (2007). Effects of thin fin on natural convection in porous triangular enclosures. International Journal of Thermal Sciences 46(10): 10331045. https://doi.org/10.1016/j.ijthermalsci.2006.11.001

[11] Varol Y, Oztop HF, Pop I. (2009). Natural convection in a diagonally divided square cavity filled with a porous medium. International Journal of Thermal Sciences 48(7): 1405-1415. https://doi.org/10.1016/j.ijthermalsci.2008.12.015

[12] Hsiao SW, Chen CK. (1994). Natural convection heat transfer from a corrugated plate embedded in an enclosed porous medium. Numerical Heat Transfer 25(3): 331345. https://doi.org/10.1080/10407789408955952

[13] Sathiyamoorthy M, Narasimman S. (2011). Control of flow and heat transfer in a porous enclosure due to an adiabatic thin fin on the hot wall. Transport in Porous Media 89(3): 421. https://doi.org/10.1007/s11242-0119778-x 
[14] Al-Makhyoul ZM. (2009). Study the effect of non-Darcy flow on natural convection inside rectangular cavity filled with saturated porous medium heated from below using two adiabatic partitions. Al-Qadisiyah Journal for Engineering Sciences 2(2): 50-69.

[15] Rees DAS, Bassom AP, Genç G. (2014). Weakly nonlinear convection in a porous layer with multiple horizontal partitions. Transport in Porous Media 103(3): 437-448. https://doi.org/10.1007/s11242-014-0310-y

[16] Rees D. (2016). Nonlinear convection in a partitioned $\begin{array}{llll}\text { porous } & \text { layer. } & \text { Fluids }\end{array}$ https://doi.org/10.3390/fluids1030024

[17] Chordiya JS, Sharma RV. (2018). Numerical study on the effects of multiple internal diathermal obstructions on natural convection in a fluid-saturated porous enclosure. Archive of Mechanical Engineering 65(4): 553-578. https://doi.org/10.24425/ame.2018.125442

[18] Bejan A, Poulikakos D. (1984). The nondarcy regime for vertical boundary layer natural convection in a porous medium. International Journal of Heat and Mass Transfer 27(5): 717-722. https://doi.org/10.1016/00179310(84)90141-8

[19] Sinha MK, Sharma RV. (2013). Natural convection in a spherical porous annulus: The brinkman extended Darcy flow model. Transport in Porous Media 100(2): 321-335. https://doi.org/10.1007/s11242-013-0218-y

[20] Sinha MK, Sharma RV. (2016). Numerical study of natural convection in a spherical porous annulus. Journal of Porous Media 19(3): 277-286. https://doi.org/10.1615/JPorMedia.v19.i3.70

[21] Mishra AK, Kumar S, Sharma RV. (2016). Non-Darcy effects on three-dimensional natural convection in a rectangular box containing a heat-generating porous medium. Journal of Porous Media 19(12): 1033-1043. https://doi.org/10.1615/JPorMedia.v19.i12.20

[22] Chordiya JS, Sharma RV. (2018). Conjugate natural convection in a fluid-saturated porous enclosure with two solid vertical partitions. Heat Transfer-Asian Research 47(8): 1031-1047. https://doi.org/10.1002/htj.21364

[23] Subhash CJ, Sharma RV. (2018). Natural convection in inclined rectangular porous enclosure with diathermal partition wall. Carbon: Science and Technology 10(1): 13-18.

[24] Walker KL, Homsy GM. (1978). Convection in a porous cavity. Journal of Fluid Mechanics 87(3): 449-474. https://doi.org/10.1017/S0022112078001718

[25] Bejan A. (1979). On the boundary layer regime in a vertical enclosure filled with a porous medium. Letters in Heat and Mass Transfer 6(2): 93-102. https://doi.org/10.1016/0094-4548(79)90001-8
[26] Manole DM. (1992). Numerical benchmark results for natural convection in a porous medium cavity. In Heat and Mass Transfer in Porous Media 216: 55-60.

[27] Moya SL, Ramos E, Sen M. (1987). Numerical study of natural convection in a tilted rectangular porous material. International Journal of Heat and Mass Transfer 30(4): 741-756. https://doi.org/10.1016/0017-9310(87)90204-3

[28] Baytas AC, Pop I. (1999). Free convection in oblique enclosures filled with a porous medium. International Journal of Heat and Mass Transfer 42(6): 1047-1057. https://doi.org/10.1016/S0017-9310(98)00208-7

\section{NOMENCLATURE}

\begin{tabular}{|c|c|}
\hline$A R$ & Aspect ratio \\
\hline$c_{p}$ & specific heat, J. $\mathrm{kg}^{-1} \cdot \mathrm{K}^{-1}$ \\
\hline$D$ & Distance of partition from origin, $m$ \\
\hline$g$ & gravitational acceleration, $\mathrm{m} \cdot \mathrm{s}^{-2}$ \\
\hline$k$ & thermal conductivity, W.m-1 $\mathrm{K}^{-1}$ \\
\hline$K$ & Permeability of porous medium $\left(\mathrm{m}^{2}\right)$ \\
\hline$L$ & Length of enclosure, $\mathrm{m}$ \\
\hline$N u$ & Nusselt number \\
\hline$P R$ & Partition Ratio \\
\hline$R a^{*}$ & Darcy modified Rayleigh number \\
\hline$T$ & Temperature, $\mathrm{K}$ \\
\hline$U, V$ & Dimensionless $\mathrm{x}$ - and $\mathrm{y}$ - velocity \\
\hline$u, v$ & $\mathrm{x}$ - and $\mathrm{y}$ - direction velocity $\left(\mathrm{m}^{2} / \mathrm{s}\right)$ \\
\hline$x, y$ & Horizontal and vertical directions (m) \\
\hline$X, Y$ & $\begin{array}{l}\text { Dimensionless horizontal and vertical } \\
\text { directions }\end{array}$ \\
\hline
\end{tabular}

\section{Greek symbols}

$\begin{array}{ll}\alpha & \text { thermal diffusivity, } \mathrm{m}^{2} \cdot \mathrm{ss}^{-1} \\ \beta & \text { thermal expansion coefficient, } \mathrm{K}^{-1} \\ \Theta & \text { dimensionless temperature } \\ \mu & \text { dynamic viscosity, } \mathrm{kg} \cdot \mathrm{m}^{-1} \cdot \mathrm{s}^{-1} \\ v & \text { Kinematic viscosity }\left(\mathrm{m}^{2} / \mathrm{s}\right) \\ \rho & \text { Density }\left(\mathrm{kg} / \mathrm{m}^{3}\right) \\ \psi & \text { Dimensionless stream function } \\ \omega & \text { Acceleration factor }\end{array}$

\section{Subscripts}

$\begin{array}{ll}\text { avg } & \text { average } \\ \mathrm{c} & \text { cold } \\ \mathrm{e} & \text { effective } \\ \mathrm{h} & \text { hot } \\ \max & \text { maximum } \\ \mathrm{p} & \text { porous }\end{array}$

